\title{
Parametric uncertainty or hydrological changes?
}

\author{
F. VIOLA, L. V. NOTO \& D. PUMO \\ Dipartimento di Ingegneria Civile, Ambientale, Aerospaziale e dei Materiali, Università degli Studi di Palermo, Italy \\ francesco.viola77@unipa.it
}

\begin{abstract}
The model calibration is the way of hydrologists for searching also a physical interpretation of complex interactions acting within a basin. Actually, it can be frequently noticed how model calibration performed on a given time-window may converge to a point in the parameter space that could be distant from another obtainable calibration of the model in the same basin but considering a different time window. Is that again parametric uncertainty or does the trajectory in the parametric space relate about to a slow hydrological basin change? This paper depicts a possible path for detecting changes' signatures in a streamflow time series. In particular, the paper seeks to draw a way to discern the random variability over different time-windows of the calibrated model parameters set from that induced by the variation in time of some boundary conditions and external forcings. To this purpose, we will refer to a conceptual lumped model for simulating daily streamflow, the EHSM (EcoHydrological Streamflow Model), and to a hypothetical case study. The selected hydrological model requires a total of seven parameters, some of which can be easily related to land use, while others rely on climate variables. The calibration of the EHSM parameters with regard to different timewindows and the analysis of potential impacts of the anthropic variation in land use and/or climatic variability on the calibrated parameters set, will support our investigation.
\end{abstract}

Key words coupled human-water systems; modelling; hydrological change

\section{INTRODUCTION}

The rapid increase of the socio-economic level in many countries has an effect on the water cycle, which is rapidly changing across the planet. Water demands increased human exploitation of water resources, modified landscapes, and influenced water cycle dynamics from local to global scales (Falkenmark \& Lannerstad 2005, Rockstrom et al. 2009, Vorosmarty et al. 2010). In order to achieve a better comprehension of hydrological systems in changing conditions and forcings, also related to societal development, the International Association of Hydrological Sciences (IAHS) has launched the Panta Rhei, a new Science Plan for the decade 2013-2022.

Abandoning the traditional hydrological perspective founded on the assumption of static systems, the new science plan wants to explore hydrological systems where human-induced modifications and natural changes are in some way coupled and interlinked. The assumption of static systems in hydrological analysis has been effective to solve water resources management and engineering problems for a long time. However, there is now a paramount need to connect environmental changes with societal changes, including the water cycle in the modelling system as an evolving component, which is directly linked with many feedbacks to humans and the Earth system. Of course to do that an interdisciplinary approach involving social scientists, economists, decision makers and users is needed.

Traditionally, change has been modelled by identifying and calibrating hydrological models in current conditions and then running them with perturbed parameters or input data according to assumptions on changes in forcings and/or dynamics. This approach has proved to be affected by significant limitations due to the limited credibility of current models under changing conditions, uncertainty in future trajectories and model parameters. The IAHS Science Plan calls for improved approaches, based on modern monitoring systems and computing facilities, aimed at understanding the interaction between hydrological systems and socio-economic-environmental change forcings.

At the same time, the IAHS Science Plan indicates that innovative ideas to extract information from the existing data are worthy of consideration as many signatures in the data are still to be explored. This last aspect is the concept behind this work, namely the possibility of detecting changes within a basin from historical streamflow data through the use of a simple conceptual hydrological model.

Under the hypothesis of the existence of a two-way relationship between model parameters and basin morphology and or climatic conditions, supposing no model and data uncertainties, this work 
is aimed at suggesting a methodology for investigating parameter evolution over time, and for exploring their possible link with tangible changes.

The duality between parametric uncertainty and boundary conditions changes is not a specific topic for a particular model, but it is a general aspect regarding the majority of the existing models, also in fields different from hydrology. In the same manner, the methodology that we are going to investigate should be considered as generic and not specific for a particular hydrological model, even if, for a better understanding of the idea behind this paper we refer to a specific model. In particular, the hydrological model here used is the EHSM (EcoHydrological Streamflow Model), a simple conceptual lumped model for simulating daily streamflow (Viola et al. 2013), with only seven parameters describing soil, vegetation and hydrological basin response.

\section{ECOHYDROLOGICAL STREAMFLOW MODEL AND FEATURES OF CASE STUDY}

The choice of a conceptual model has been driven by the advantages offered at the same time by model simplicity and parameter physical meaning. The simple model structure conceptualizes some basin hydrological properties and mechanisms such as soil permeability, vegetation presence together with its role in water uptake, and groundwater response. The hydrological model consists of three interconnected elements: a soil bucket and two linear reservoirs. The soil bucket distinguishes two soils classes, the first interpreting totally impermeable soils, while the second one describes permeable soils characteristics.

The surface soil bucket is linked to the two linear reservoirs: one is responsible for the fast surface runoff component, while the other accounts for the slow subsurface runoff component. The surface reservoir is fed by the rain falling on impermeable areas and by the saturation excess generated over the permeable area. These two mechanisms are characterized by comparable times of response and together contribute to the fast component of runoff. The fast component is assumed to be instantaneously transferred to the surface reservoir, which is characterized by a mean residence time having the same order of magnitude as the basin concentration time. The subsurface reservoir, solely supplied by leakage pulses, is typically characterized by longer response times.

The soil schematization in an impermeable fraction $c_{0}$ and a permeable fraction $\left(1-c_{0}\right)$ where vegetation is located and water can infiltrate, allowing a description of the temporal evolution of the urbanization and the consequent depletion of agricultural/natural landscapes, which could take place during some decades.

The permeable fraction of soil is characterized by the soil porosity and by the root depth, termed $n$ and $Z_{r}$, respectively, whose product, known also as "active soil depth", is here considered as a unique parameter. This characteristic has a slow dynamic related to the soil formation and to the vegetation pattern evolution, both millenarian.

Daily rainfall depth $R$, considered as the main climatic forcing, is provided to the model as a time series spatially averaged over the catchment. That explicitly includes the climatic interannual variability in the model, which is crucial for the Mediterranean context where, in addition to the marked seasonality (Bolle 2003), decreasing rainfall trends have been observed (Cannarozzo et al. 2006). Rainfall amount falling over the impervious area, $R_{i m p}$, is transferred to the surface linear reservoir, while the amount falling on the pervious part of the soil, termed $R_{\text {perm }}$, drives relative soil moisture dynamics. The determination of relative soil moisture variation $\Delta s$ in the permeable part of the soil bucket during a daily temporal step, is achieved with the numerical solution of the following water balance equation, carried out through a forward finite differences method (Viola et al. 2013):

$$
\frac{\Delta s}{\Delta t}=\frac{1}{n Z_{r}\left(1-c_{0}\right)}\left(R_{p e r m}-Q_{s}-E T-L\right)
$$

where $Q_{s}$ is the runoff over the permeable part of the basin, and $E T$ and $L$ represent the soil water losses due to evapotranspiration and leakage. The relationship between the rate of plant water uptake and the soil moisture is often modelled as a stepwise function (Laio et al. 2001, Pumo et al. 2008, Viola et al. 2008). In the EHSM the evapotranspiration-leakage-soil moisture relation, is simplified 
by assuming the presence of a soil moisture value, named $s_{t}$, over which evapotranspiration occurs at the maximum rate $E_{\max }$. When water is accumulated between $s_{t}$ and the saturation point $(s=1)$, it is lost instantaneously as leakage, feeding the subsurface reservoir. Below $s_{t}$, evapotranspiration is reduced linearly to zero at a point named $s_{0}$, which can be defined as a hygroscopic point. These soil moisture values are physically related to texture and vegetation physiology, which evolve slowly in time and space.

The relationship providing water losses from the permeable soil as a function of soil moisture thus reads:

$$
E T+L=\left\{\begin{array}{lc}
0 & \text { if } s \leq s_{0} \\
E_{\max }\left(\frac{s-s_{0}}{s_{t}-s_{0}}\right)\left(1-c_{0}\right) & \text { if } s_{0}<s \leq s_{t} \\
E_{\max }\left(1-c_{0}\right)+\left(s-s_{t}\right) n Z_{r}\left(1-c_{0}\right) & \text { if } s_{t}<s \leq 1
\end{array}\right.
$$

The daily evapotranspiration rate in well-watered conditions, $E_{\max }$, is evaluated using the FAO-56 Penman-Monteith method (Allen et al. 1998) as a function of solar radiation, air temperature, humidity and wind speed. The EHSM introduced a vegetational coefficient, $K_{v}$, with the aim to feature virtual vegetation, lying over the permeable part of the basin, which is conceptually similar to the crop coefficient. The method used to calculate potential daily evapotranspiration rate allows the implicit accounting for climate variability, as for example the temperature increase (Viola et al. 2014), while preserving the capability of describing the vegetation influence on the basin water balance. Even if vegetation patterns change slowly, human-induced transformations like deforestation or agricultural settlement may be very fast, thus abruptly changing basin water demand and evapotranspirative fluxes.

The three components generating runoff at the basin outlet, namely the runoff from impervious surface, $Q c_{0}$, the excess saturation surface runoff, $Q_{s}$, and the leakage $L$, are routed into two classic linear reservoirs. Water contributions arising from the top layer of the soil, namely $Q c_{0}$ and $Q_{s}$, are diverted into a surface linear reservoir which is characterized by a residence time $1 / K_{\text {sup }}$, while the leakage contribution fed a subsurface reservoir, characterized by a residence time $1 / K_{\text {sub }}$. Both the reservoirs have an outflow, $Q_{s u p}$ and $Q_{s u b}$, respectively, which are a linear function of water stored in the superficial and in the subsurface reservoir. The surface reservoir aims to describe the quick hydrological basin response, thus the residence time $1 / K_{\text {sup }}$ is expected to be of the same order of magnitude as the basin concentration time. Conversely, the subsurface reservoir is aimed at describing the slow hydrological basin response, and, consequently, the residence time $1 / K_{\text {sub }}$ is larger than $1 / K_{\text {sup }}$. The dependency of the reservoirs' constants from the underlying climatic conditions has been proven (Pumo et al. 2013), and there is a clear link between these conceptual parameters and the basin morphology (Post and Jakeman 1996). In this sense, the residence times also appear to be suitable for investigating the inter-dependence between climatic variability and the hydrological response.

A possible, challenging, case study could be a Mediterranean catchment, because during the last century, several basins have undergone an urbanization increase, mostly focused in the 70th and 80th. At the same time, these changes have been accompanied by a reduction of natural resources, basically due to the rainfall reduction (Kutiel et al. 1996, Piervitali et al. 1997, Cannarozzo et al. 2006).

The feasibility of such a study is conditioned to a wide availability of climatic data. In particular, for the model calibration rainfall, temperature and streamflow data are strictly necessary for calibrating the EHSM parameters in different time-windows. Further records of solar radiation, wind speed, and relative humidity would allow a more accurate estimation of reference evapotranspiration. At the same time a deep knowledge of human activity over the years within the basin is necessary. The knowledge of a hypothetical case study could be built, for example, using different sources of information such as orthophotos or Landsat MSS and TM satellite images. Different studies have already been carried out using these sources of data for extracting land use/cover change 
data from 1975 to nowadays in the Brasilian Amazonia, Costa Rica, Mediterranean areas, West Africa, China, United States (Lambin and Strahler 1994, Carlson and Sanchez-Azofeifa 1999, Carlson \& Arthur, 2000, Weng 2002, Yang and Lo 2002, Cardille and Foley 2003, Deng et al. 2008, Hill et al. 2008, Zhang et al. 2009, Liu and Tian 2010). Also regional water permits and census data could give an historical view on land uses, with particular reference to urban areas increase, vegetation patterns and water abstractions.

\section{ROADMAP FOR FUTURE INVESTIGATION}

An automatic parameter calibration procedure will be carried out using the Monte Carlo method (Metropolis \& Ulam 1949). The method consists in the random generation of several parameter vectors $\theta_{i}=\left(n Z_{r}, s_{t}, s_{0}, c_{0}, K_{v}, K_{\text {sup }}, K_{\text {sub }}\right)$ and then, in the evaluation of the model performance in reproducing historical streamflow series associated with each tested set of parameters, through an appropriate likelihood measure. Model parameters will be chosen within wide ranges according to a uniform distribution, with the extremes of such ranges fixed on the basis of a specific knowledge of the case study. The performance of each individual parameter set will be assessed using the Nash and Sutcliffe (NS) efficiency criterion (Nash 1970) based on the sum of squared errors:

$$
\operatorname{NSE}\left(\theta_{i} / Y\right)=\left(1-\sigma_{i}^{2} / \sigma_{o b s}^{2}\right) \quad \sigma_{i}^{2}<\sigma_{o b s}^{2}
$$

where $\operatorname{NSE}\left(\theta_{i} / Y\right)$ is the likelihood measure for the $i$ th model simulation for parameter vector $\theta_{i}$ conditioned on a set of observations $Y, \sigma_{i}^{2}$ is the associated error variance for the $i$ th model and $\sigma_{o b s}{ }^{2}$ is the observed variance for the given period.

From the historical series available some continuous sub-series for simulations will be defined. Several calibrations will be carried out using, for each time-window, daily series of rainfall, discharge and reference evapotranspiration. Ten-year series with an overlapping of 5 years in respect to both the previous and the following time window will be considered.

Several models are expected to lead to an equally acceptable representation of the observed hydrological processes within a given sampling period. This assertion is justified by previous model calibration on a Mediterranean basin that showed a certain degree of parameters uncertainty (Viola et al. 2013). In order to manage this parametric uncertainty (Beven and Freer 2001) the highest model efficiencies (i.e. above the 99th percentile) will be considered, together with the associated parameter vector $\theta_{i}$. This will allow provision of a posteriori parameters distributions: the ensemble of these distributions will be considered as the hydrological model response for a given time window.

At the same time, focusing the attention on the a posteriori parameters distribution, some modifications over time could occur. The a posteriori parameter distributions "rehashing" (computable in terms of moments of different order) could be interpreted again as parameter uncertainty or as a hydrological basin signature evolving during the last century in response to several climatic or anthropic changes. The distinction between these alternatives is linked to the possibility of functionally explaining the a posteriori distribution evolution with the soil and vegetation transformations and/or with human interactions and/or climatic variability. Whereas these relations could be not explained by land-use changes or climatic variability, parameters oscillation should be only attributed to randomness.

Functional relationships will be investigated between each model parameter (in terms of expected value, variance, etc. of the a posteriori distribution) and basin morphologic attributes and/or climatic conditions (estimated or measured in each sub-period) using multiple regression analyses. Hereafter some investigation direction and expected situations are depicted on the basis of a potential application of the EHSM model.

The active soil depth parameter $\left(n Z_{r}\right)$, which is related to soil porosity and vegetation rooting depth, the soil moisture value triggering leakage $\left(s_{t}\right)$, the soil moisture value $\left(s_{0}\right)$ which stops evapotranspiration, and the model parameter responsible for scaling the reference evapotranspiration $\left(K_{v}\right)$ are expected to slowly change because soil characteristics and vegetation patterns dynamics are slower than the considered reference time period. Possible changes in the a posteriori parameters 
distributions could be related to human-induced transformation such as deforestation/reafforestation (quantitatively estimable in terms of forested area percentage) or agricultural land abandonment (losses of cultivated areas percentages in favour of scrub or macchia).

The residence times of the two reservoirs are worth considering separately. Although the fluctuation of the surface response $\left(K_{\text {sup }}\right)$ could be considered irrelevant in determining model performances, because often the basin time lag is inferior to the model temporal step, the residence time of the subsurface reservoir $\left(K_{\text {sub }}\right)$ is crucial in determining the basin response. This parameter is conceptually related to the underlying climatic conditions, and in the long term, to basin morphology. Considering the elevated climatic interannual variability and the presence of trends (Cannarozzo et al. 2006, Viola et al. 2013) it will be necessary to analyse their possible influence on this parameter. This inter-relationship could be investigated using a basic climatic descriptor, as the mean rainfall frequency and reference evapotranspiration (Pumo et al. 2013), easily retrievable from historical data.

Finally, it will be worth considering the role of the impervious soil fraction $\left(c_{0}\right)$, which is the model parameter most related to the short-term human activities within a basin. It is expected that urbanization drives the evolution of this parameter, or in other words that increasing the fraction of impervious area with roads, roofs and flooring, this model parameter responds in a coherent way. The fraction of impervious area could be obtainable from Landsat images from 1975 onwards and relatable to the number of inhabitants and/or to the areal gross income. This will also allow inference of the $c_{0}$ value before the satellite images era.

\section{CONCLUSIONS}

The interpretation of changes in hydrological systems is one of the most interesting emerging research topics. The IAHS has declared the possibility of reaching an improved interpretation of the processes governing the water cycle as a scientific objective by focusing on their changing dynamics, in connection with rapidly changing human systems. The classical view that treats the catchment as a fixed entity should be overcome by new approaches that take into account the coevolution of hydrological, geomorphologic, chemical and ecological systems.

This work tracks a possible way to explore hydrological changes from the streamflow data signature. A possible methodology to discern between parametric uncertainty and hydrological response changes is sketched here through the discussion of a potential application of the simple conceptual model, EHSM. Calibrating the model over different consecutive time windows, different behaviours could emerge. This could be interpreted as a different hydrological response to different climatic forcings and basin boundary conditions (soil and vegetation patterns) or as parametric uncertainty. Distinguishing between these two cases is not simple: several factors, such as model or data uncertainties, may act as catalyst of parametric uncertainty. It is also true that the calibration procedure, which is conceptually carried out regardless of the physical meaning of model parameters and retains some maxima of the local likelihood, is prone to parametric uncertainty.

Rather than on a single "optimal" calibration set, the analysis of the vectors containing the calibrated parameters set for each considered time window, should be performed on the a posteriori distributions of the parameters associated to the highest model efficiencies (for example, NSE above the 99th percentile), studying, for example, changes in the moments of various order of the distribution.

Under the hypothesis of a link between model parameters and basin climatic/morphologic descriptors, it is argued that a hydrological response change of the a posteriori parameter distribution change could be functionally linked with observed modifications or alterations. Otherwise, parameter oscillation over different calibration periods should be considered just as parametric uncertainty.

\section{REFERENCES}

Allen, R. G., et al. (1998) Crop evapotranspiration-Guidelines for computing crop water requirements. FAO Irrigation and drainage paper 56. FAO, Rome 300, 6541 .

Beven, K. and Freer, J. (2001) Equifinality, data assimilation, and uncertainty estimation in mechanistic modelling of complex environmental systems using the GLUE methodology. Journal of Hydrology 249(1-4), 11-29. 
Bolle, H. J. (2003) Mediterranean Climate. Springer

Cannarozzo, M., Noto, L. V., and Viola, F. (2006) Spatial distribution of rainfall trends in Sicily (1921-2000). Physics and Chemistry of the Earth 31(18), 1201-1211.

Cardille, J. A. and Foley, J. A. (2003) Agricultural land-use change in Brazilian Amazonia between 1980 and 1995: Evidence from integrated satellite and census data. Remote Sensing of Environment 87(4), 551-562.

Carlson, T. N. and Sanchez-Azofeifa, G. A. (1999) Satellite remote sensing of land use changes in and around San Jose, Costa Rica. Remote Sensing of Environment 70(3), 247-256.

Carlson, T. N. and Arthur, S. T. (2000) The impact of land use - land cover changes due to urbanization on surface microclimate and hydrology: a satellite perspective. Global and Planetary Change 25(1-2), 49-65.

Deng, J. S., et al. (2008) PCA-based land-use change detection and analysis using multitemporal and multisensor satellite data. International Journal of Remote Sensing 29(16), 4823-4838.

Falkenmark, M. and Lannerstad, M., (2005) Consumptive water use to feed humanity - curing a blind spot. Hydrology and Earth System Sciences $9(1-2), 15-28$.

Hill, J., et al. (2008) Mediterranean desertification and land degradation Mapping related land use change syndromes based on satellite observations. Global and Planetary Change 64(3-4), 146-157.

Kutiel, H., Maheras, P. and Guika, S. (1996) Circulation and extreme rainfall conditions in the eastern Mediterranean during the last century. International Journal of Climatology 16(1), 73-92.

Laio, F., et al. (2001) Plants in water-controlled ecosystems: active role in hydrologic processes and response to water stress -II. Probabilistic soil moisture dynamics. Advances in Water Resources 24(7), 707-723.

Lambin, E. F. and Strahler, A. H. (1994) Change-vector analysis in multitemporal space - a tool to detect and categorize landcover change processes using high temporal-resolution satellite data. Remote Sensing of Environment 48(2), 231-244.

Liu, M. and Tian, H. (2010) China's land cover and land use change from 1700 to 2005: Estimations from high-resolution satellite data and historical archives. Global Biogeochemical Cycles 24.

Metropolis, N. and Ulam, S. (1949) The Monte Carlo Method. Journal of the American Statistical Association 44(247), 335-341.

Nash, J. E. and Sutcliffe, J. V. (1970) River flow forecasting through conceptual models, 1. A discussion of principles. Journal of Hydrology 10, 282-290.

Piervitali, E., Colacino, M. and Conte, M., (1997) Signals of climatic change in the central-western Mediterranean basin Theoretical and Applied Climatology 58(3-4), 211-219.

Post, D. A. and Jakeman, A. J. (1996) Relationships between catchment attributes and hydrological response characteristics in small Australian mountain ash catchments. Hydrological Processes 10(6), 877-892.

Pumo, D. and Noto, L. V. (2013) EHSM: A conceptual ecohydrological model for daily streamflow simulation. Hydrological Processes, Hydrological Processes 2013. DOI: 10.1002/hyp.9876.

Pumo, D., Viola, F. and Noto, L. V. (2008) Ecohydrology in Mediterranean areas: a numerical model to describe growing seasons out of phase with precipitations. Hydrol. Earth Syst. Sci. 12(1), 303-316.

Pumo, D., Noto, L. V. and Viola, F. (2013) Ecohydrological modelling of flow duration curve in Mediterranean river basins. Advances in Water Resources 52, 314-327.

Rockstrom, J., et al. (2009) Planetary Boundaries: Exploring the Safe Operating Space for Humanity. Ecology and Society 14(2).

Viola, F., et al. (2008) Transient soil-moisture dynamics and climate change in Mediterranean ecosystems. Water Resources Research 44(11).

Viola, F., et al. (2014) Spatial distribution of temperature trends in Sicily. International Journal of Climatology 34(1).

Vorosmarty, C. J., et al. (2010) Global threats to human water security and river biodiversity (vol 467, pg 555, 2010). Nature 468(7321), 334-334

Weng, Q. H. (2002) Land use change analysis in the Zhujiang Delta of China using satellite remote sensing, GIS and stochastic modelling. Journal of Environmental Management 64(3), 273-284.

Yang, X. and Lo, C. P. (2002) Using a time series of satellite imagery to detect land use and land cover changes in the Atlanta, Georgia metropolitan area. International Journal of Remote Sensing 23(9), 1775-1798.

Zhang, J., Liu, Z., and Sun, X. (2009) Changing landscape in the Three Gorges Reservoir Area of Yangtze River from 1977 to 2005: Land use/land cover, vegetation cover changes estimated using multi-source satellite data. International Journal of Applied Earth Observation and Geoinformation 11(6), 403-412. 